\title{
Biomarkers for the detection of apparent and subclinical cancer therapy-related cardiotoxicity
}

\author{
Lars Michel, Tienush Rassaf, Matthias Totzeck \\ Department of Cardiology and Vascular Medicine, West German Heart and Vascular Center, Medical Faculty, University Hospital Essen, Essen, \\ Germany \\ Contributions: (I) Conception and design: L Michel, M Totzeck; (II) Administrative support: T Rassaf, M Totzeck; (III) Provision of study materials \\ or patients: None; (IV) Collection and assembly of data: L Michel, M Totzeck; (V) Data analysis and interpretation: All authors; (VI) Manuscript \\ writing: All authors; (VII) Final approval of manuscript: All authors. \\ Correspondence to: Dr. Matthias Totzeck, MD. Department of Cardiology and Vascular Medicine, West German Heart and Vascular Center, Medical \\ Faculty, University Hospital Essen, Hufelandstraße 55, 45147 Essen, Germany. Email: Matthias.Totzeck@uk-essen.de.
}

\begin{abstract}
Progress in cancer therapy over the past decades improved long-term survival but increased cancer therapy-related cardiotoxicity. Many novel treatment options have been implemented with yet incompletely characterized cardiovascular side effects including heart failure, coronary artery disease, arrhythmias, valvular disease, venous thromboembolism and myocarditis. Diagnosis of potential cardiotoxic side effects is essential for an optimal treatment but remains challenging. Cardiac biomarkers troponin and brain natriuretic peptide/N-terminal proBNP (BNP/NT-proBNP) have been extensively studied in heart failure and acute coronary syndromes. Emerging evidence implicates a significant role in the detection of cardiotoxicity and guidance of therapy in cancer patients. Elevated troponin or BNP/NT-proBNP levels were associated with increased all-cause mortality in cancer patients and have been shown to predict manifest heart failure. BNP/NT-proBNP may be useful for the prediction of cancer therapy-related heart failure and response to heart failure therapy in adult and pediatric cancer patients while troponin can indicate acute myocardial infarction in patients with cancer therapy-related risk for coronary artery disease. Furthermore, troponin may be used for the identification of immune checkpoint inhibitor-related myocarditis with very high sensitivity. Finally, even D-dimer levels have been shown to improve risk stratification and diagnosis in cancer-associated venous thromboembolism. This review aims to summarize the current knowledge about biomarkers in cancer therapy-related cardiotoxicity. We also outline possible clinical recommendations for the detection and treatment of subclinical and clinically apparent cardiotoxic effects using biomarkers.
\end{abstract}

Keywords: Biomarker; brain natriuretic peptide (BNP); cardiotoxicity; cardio-oncology; troponin

Submitted Jul 31, 2018. Accepted for publication Aug 03, 2018.

doi: $10.21037 /$ jtd.2018.08.15

View this article at: http://dx.doi.org/10.21037/jtd.2018.08.15

\section{Introduction}

Cancer and heart disease are the leading causes of death in western civilization $(1,2)$. Progress in cancer treatments over the past decades improved long-term survival of cancer patients (3). Cardiotoxic effects of traditional therapy regimes and novel treatments have contributed to an increased cardiovascular morbidity and mortality in cancer survivors. Cardiovascular adverse events can arise from radiotherapy, cytotoxic chemotherapy, molecularly targeted inhibitors and antibodies directed against signal transduction pathways and immune checkpoints. Cardiovascular complications may present as, e.g., heart failure, coronary artery disease (CAD) and acute coronary syndrome (ACS), myocarditis, venous thromboembolism (VTE), valvular pathologies and arrhythmias (4). The incidence and course of cancer therapy-associated cardiotoxicity is particularly undefined for novel therapies 
(5-8). The emerging field of cardio-oncology aims to improve risk assessment, diagnosis and treatment of cancer therapy-induced cardiovascular disease. Specific diagnostic algorithms are particularly important for risk stratification, monitoring and long-term follow-up of cancer patients $(4,7,9)$. Biomarkers represent a mainstay for the diagnosis of acute and chronic heart disease. Their role for the timely detection and monitoring of cardiotoxicity is, however, incompletely characterized and appears more complex than in primary cardiovascular diseases (4). This review aims to summarize the current evidence, recommendations and future perspectives regarding biomarker-guided diagnosis and therapy of cancer therapy-related cardiovascular adverse events.

\section{Biomarkers in cardiovascular medicine}

Biomarkers have been established for diagnosis and treatment of many cardiovascular diseases. Brain natriuretic peptide (BNP) and N-terminal proBNP (NT-proBNP) are the canonical biomarkers for the detection of heart failure (10-12). BNP is synthesized and secreted by cardiomyocytes in response to transmural pressure or neurohumoral stimulation (noradrenaline, angiotensin II) (13). Following synthesis of preproBNP and removal of a 25 amino acid residue, the prohormone proBNP can be stored in intracellular vesicles. During activation, proBNP is cleaved into the active BNP and biologically inactive NT-proBNP. Both peptides are secreted in equimolar amounts with BNP half-life around $20 \mathrm{~min}$ and that of NT-proBNP at around 120 min resulting in a 4-6-fold higher NT-proBNP plasma concentration $(13,14)$. BNP is biologically active (13). Elevated levels decrease cardiac preload and afterload by increasing glomerular filtration rate (GFR) and vasodilation. Furthermore, BNP inhibits unfavorable myocardial remodeling. This balances maladaptive neuro-humoral mechanisms in heart failure $(13,15)$. BNP and NT-proBNP signal heart failure with a very high sensitivity yet low specificity (10-12). The lower diagnostic limit for BNP is $35 \mathrm{ng} / \mathrm{mL}$ and for NT-proBNP $125 \mathrm{ng} / \mathrm{mL}$ [negative predictive value for heart failure of 0.94-0.98 with a positive predictive value of $0.44-0.57,(11)]$. According to European Society of Cardiology (ESC) and American Heart Association (AHA) guidelines, BNP/NTproBNP measurement is therefore a heart failure criterion (10-12). Also, in patients at risk of developing heart failure, BNP screening may be useful for early treatment intervention to prevent progression (10-12). Notably, the role of BNP/NT-proBNP in biomarker-guided therapy in patients with established heart failure remains controversial (16).

Many diseases and particularly renal failure contribute to an increase in natriuretic peptides. BNP and NT-proBNP are eliminated from plasma by several specific pathways (14): when BNP binds to BNP-receptors, it is cleaved by an endopeptidase or excreted from the kidney. In contrast, NT-proBNP is completely excreted by the kidney. While plasma concentrations of both peptides are increased in renal insufficiency, the NT-proBNP/BNP ratio correlates inversely with the estimated GFR. Therefore, NT-proBNP and BNP plasma levels should always be interpreted in the context of the renal function indicated by GFR (14). In addition, age, female sex, hypertension, atrial fibrillation and diabetes mellitus have been associated with elevated BNP/NT-proBNP levels. In the field of cancer and cancer therapy, anemia and fluid are particularly prone to impact natriuretic peptide levels $(13,17)$. Interestingly, obesity shows an inverse relationship with BNP levels. Baseline BNP was nearly bisected in patients with a body mass index (BMI) of $>30 \mathrm{~kg} / \mathrm{m}^{2}$ compared to $\mathrm{BMI}<25 \mathrm{~kg} / \mathrm{m}^{2}$ controls. This effect is possibly mediated by a higher GFR in obese patients and a reduction of circulating BNP by enhanced binding of BNP by adipocytes (18).

Cardiac troponin is the primary biomarker for diagnosis of an acute myocardial infarction given its excellent specificity and sensitivity $(19,20)$. Troponin C, troponin I and troponin $\mathrm{T}$ form a complex that is bound to tropomyosin in the contractile apparatus of cardiomyocytes (21). Troponin $\mathrm{C}$ can bind intracellular calcium, which then causes a conformational change removing tropomyosin from the myosin binding-site. This enables myosin to bind actin for cardiomyocyte contraction (21). Damage of myocardial cells causes a rise of troponin I and $\mathrm{T}$ systemic blood concentrations with a peak serum concentration $12 \mathrm{~h}$ after myocardial injury $(21,22)$. In patients with suspected non-ST-elevation ACS (NSTEACS), elevated serum high-sensitive cardiac troponin at the time of admission or at re-test above the $99^{\text {th }}$ percentile of healthy individuals indicates myocardial infarction (19-21). Over the past decade, troponin detection assays have been optimized (high/ultra-sensitive troponin) (21). This has reduced the necessary time for diagnosis of an acute myocardial infarction to a maximum of 3 hours according to ESC and AHA guidelines (19-21). Several prospective studies demonstrated a negative predictive value $>99 \%$ in high-sensitive troponin I measurements 1 hour after onset of symptoms (19,20,23-26). However, serum troponin can also be chronically elevated 


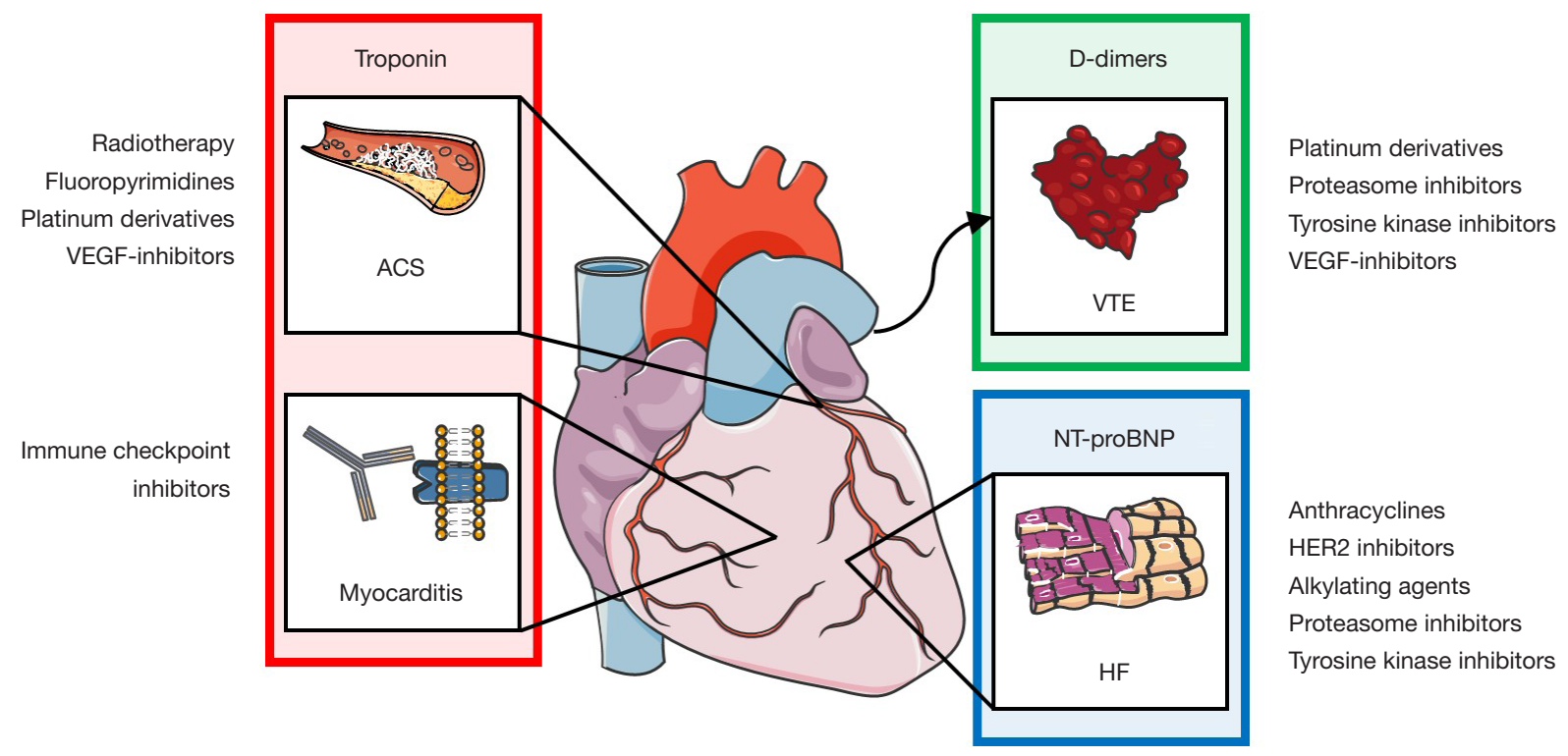

Figure 1 Biomarkers in different forms of cancer therapy-related cardiotoxicity. Various forms of cancer therapy exhibit cardiotoxic side effects. Troponin may serve as a marker for acute coronary syndrome in patients receiving radiotherapy, fluoropyrimidines, platinumbased antineoplastic drugs or vascular endothelial growth factor receptor inhibitors. In addition, assessment of serum troponin in suspected immune checkpoint inhibitor-related myocarditis can be recommended. D-dimer levels may indicate cancer- or cancer therapy-related venous thromboembolism. BNP/NT-proBNP is associated with cancer therapy-related heart failure induced by anthracyclines, HER2 inhibitors, alkylating agents, tyrosine kinase inhibitors and proteasome inhibitors. This figure was created using SMART Servier Medical Art templates (https://smart.servier.com/). ACS, acute coronary syndrome; VEGF, vascular endothelial growth factor receptor; VTE, venous thromboembolism; NT-proBNP, N-terminal pro brain natriuretic peptide; HF, heart failure; HER2, human epidermal growth factor receptor 2 .

in certain cardiovascular diseases, e.g., heart failure, stable CAD and chronic kidney disease. In these cohorts, elevated troponins serve as an independent marker for mortality and should prompt further cardiovascular disease follow-up $(21,27)$. Although troponin levels above the $99^{\text {th }}$ percentile are most relevant for the detection of disease and prognosis, recent data implicate a relevance for values above the detection limit but below the $99^{\text {th }}$ percentile: In patients presenting with acute chest pain any detectable serum troponin $\mathrm{T}$ below the $99^{\text {th }}$ percentile was associated with a 2-fold increased all-cause mortality, increased cardiovascular mortality and increased non-cardiovascular mortality (28).

Taken together, biomarkers have helped to improve diagnosis and prognostic assessment in cardiovascular diseases leading to improved patient care.

\section{Biomarkers for cardiovascular disease in cancer patients}

Based on the available evidence, only a few recommendations for the use of biomarkers in clinical cardio-oncology practice are currently available (Figure 1). In the following, the current knowledge about biomarkers for cancer therapyrelated cardiotoxicities will be discussed.

\section{Heart failure}

Both cancer and cancer-therapy contribute to cardiovascular disease in cancer patients (29). Comorbidities, e.g., severe infections, anemia and thrombocytopenia can furthermore negatively influence the outcome of patients. Heart failure is the most common form of cancer therapy-induced cardiotoxicity (30). Left ventricular (LV) dysfunction and apparent heart failure can arise from radiotherapy, cytotoxic chemotherapy, molecularly targeted inhibitors and antibodies targeting signal transduction pathways (4). Anthracyclines contribute to the majority of subclinical and clinical LV dysfunction (4). The underlying pathomechanisms are complex $(6,31)$.

On average, $18-48 \%$ of patients show signs of $L V$ 
dysfunction following high cumulative dose anthracycline therapy (e.g., doxorubicin $700 \mathrm{mg} / \mathrm{m}^{2}$ ) (32). Combination therapy with chest/mediastinal radiation or HER-2 inhibitors (e.g., trastuzumab) as commonly used in breast and gastric cancer therapy can further multiply anthracycline-induced cardiotoxic effects (33-39). Cumulative dose, intravenous bolus administration, female sex, hypertension, diabetes mellitus and known cardiovascular disease are risk factors for the development of anthracycline cardiotoxicity $(5,7,32,35,40)$. In a prospective study of 2,625 patients receiving anthracycline-containing chemotherapy, the median time between end of chemotherapy and cardiotoxicity development was 3.6 months with only $11 \%$ full recovery (40). A matched-control study showed echocardiographic cardiac abnormalities in $30 \%$ of longterm survivors of childhood acute lymphoblastic leukemia (ALL) that received anthracycline-based therapy after a median time of 13.3 years (41). Several studies have indicated a potentially prophylactic and therapeutic effect of conventional heart failure therapy (e.g., $\beta$ blockers, angiotensin-receptor blockers (AT1 blocker)) in patients with anthracycline-induced heart failure. Early diagnosis is therefore essential $(36,42,43)$.

Several other common cancer drugs have been associated with the development of heart failure, e.g., alkylating agents (cyclophosphamide and ifosfamide) $(4,5)$. Heart failure occurs more acutely after therapy (within 3 weeks) in up to $28 \%$ of patients and can be associated with high mortality $(4,5,44,45)$. Furthermore, molecular targeted therapeutics can exhibit relevant cardiotoxicity (46-50). The proteasome inhibitor carfilzomib is used in multiple myeloma therapy and caused LV dysfunction in $4 \%$, hypertension in $12 \%$ and peripheral edema in up to $25 \%$ in a dose-dependent manner (51). The complete list of cancer drugs with ascribed risk of $\mathrm{LV}$ dysfunction is, however, beyond the scope of this review $(4,49,51,52)$. Biomarkers emerge as essential tools for the evaluation of cancer therapy-induced heart failure. In this setting, troponin and BNP/NT-proBNP are the two most studied biomarkers in both retrospective and prospective cardiotoxicity studies.

\section{Natriuretic peptides}

Anthracyclines and many other therapies can induce chronic heart failure. Interestingly, observational and experimental studies have yielded inconsistent results regarding the association of serum BNP levels and chronic cardiotoxicity (53). A recent meta-analysis of eight independent case-control studies with 695 anthracyclinetreated patients showed a small, yet statistically significant correlation between the onset of anthracyclineinduced cardiotoxicity and post-treatment BNP. This difference was more pronounced in Asian rather than European cohorts (53). In a prospective study in patients with primary diagnosis of cancer, NT-proBNP was a predictor of all-cause mortality (29). However, two other investigations failed to show an association between plasma NT-proBNP level and development of LV dysfunction and manifest heart failure $(54,55)$. Children undergoing cytotoxic cancer therapy are at high risk for development of LV dysfunction (41). In a randomized controlled study, 205 children with high-risk ALL were randomized to doxorubicin treatment alone or doxorubicin with cardioprotective dexrazoxane treatment (56). Children treated with doxorubicin alone showed a higher frequency of increased NT-proBNP compared to children treated with doxorubicin and dexrazoxane. Serum troponin T was elevated in $47 \%$ of doxorubicin-only treated children compared to $13 \%$ of doxorubicin and dexrazoxane-treated children and was associated with decreased $L V$ ejection fraction (LVEF) after 90 days (56). In children with hematologic malignancies treated with anthracyclines, the role of angiotensin converting enzyme inhibitors (ACEinhibitors) has been tested in a double blind, prospective trial (57). After six months, NT-proBNP in placebo-treated patients was increased from $<5 \mathrm{ng} / \mathrm{mL}$ baseline value to $98.6 \mathrm{ng} / \mathrm{mL}$. Children treated with enalapril showed a $50 \%$ lower plasma NT-proBNP of $49.6 \mathrm{ng} / \mathrm{mL}$ together with higher LVEF. These data indicate a role of BNP in monitoring cardioprotective therapy in children undergoing anthracycline-based chemotherapy. In long-term survivors of childhood cancer, two studies have been conducted investigating plasma BNP/NT-proBNP in a total of 206 patients $(58,59)$. NT-proBNP was correlated with the cumulative anthracycline dose. Furthermore, a correlation between elevated NT-proBNP and LV dysfunction was found $(58,59)$. Taken together, BNP/NT-proBNP was associated with cancer therapy-related chronic heart failure in adult and pediatric cancer patients $(53,56)$.

Acute heart failure induced by cancer therapy may be an acute life-threating condition. High-dose cyclophosphamide treatment is often used in hematologic malignancies prior to bone marrow transplantation. Administration can acutely induce severe heart failure with high mortality (45). The significant proportion of comorbidities in patients 
undergoing high-dose chemotherapy for bone marrow transplantation impedes diagnostic and therapeutic measures $(44,45)$. Therefore, screening for cardiovascular risk factors and disease prior to therapy together with monitoring for cardiovascular adverse events is crucial (60). In a small cohort of patients undergoing bone marrow transplantation, weekly BNP measurements identified all patients with therapy-associated heart failure (20\%) with a specificity of $43 \%(61)$. The authors argue that comorbidities, e.g., anemia, sepsis, fluid imbalance or renal failure could potentially downgrade the diagnostic value of BNP in this setting (61). In summary, BNP/NT-proBNP monitoring serves to identify acute cancer therapy-related heart failure (61).

\section{Troponin}

The association of elevated troponin and cancer therapyrelated cardiotoxicity has previously been studied in several cohorts (62). High-sensitive troponin $\mathrm{T}$ in patients with primary cancer diagnosis has been shown to predict overall mortality with a cutoff value of $0.005 \mathrm{ng} / \mathrm{mL}$ (29). Elevated serum troponin $\mathrm{T}$ was furthermore associated with advanced tumor stage (29). Chemotherapy protocols containing alkylating agents or anthracyclines have shown plasma troponin I elevation in $12-33 \%$ in adults $(43,54,62-65)$ and up to $47 \%$ in children (56). The cardiotoxic effect of the human epidermal growth factor receptor 2 inhibitor (HER2 inhibitor) trastuzumab with or without anthracycline-based chemotherapy has been extensively studied as it affects a significant portion of breast cancer patients $(37,38,66)$. Trastuzumab monotherapy caused elevated troponin I in 14\% while in combination therapy of trastuzumab with anthracyclines, elevated troponin I was observed in $21-24 \%$ of patients $(54,63,67)$. Notably, a comparably mild troponin I elevation below three times the upper limit was determined in these studies (43). Taken together, troponin serves to predict heart failure in patients receiving cardiotoxic cancer therapy (64).

Troponin as a marker of manifest heart failure has been investigated in different retrospective and prospective settings (55). In 204 cancer patients receiving anthracyclinebased or alkylating high-dose chemotherapy, positive troponin I was associated with marked reduction of LVEF after seven months while troponin I negative patients showed only a transient ( $<3$ months) and less marked LVEF reduction (62). In 211 high-risk breast cancer patients, an association between troponin I elevation and LVEF reduction (12\% vs. $2 \%)$ could be recapitulated over a period of 12 months (65). Plasma troponin I at the completion of anthracycline administration (maximum troponin I) and troponin I measured 3 months after therapy were predictors of cardiotoxicity $(54,55)$. Trastuzumab-induced heart failure determined by reduced LVEF was more frequent in patients with elevated troponin I (67). Only limited data is available for repetitive troponin tests in the course of cancer therapy. A prospective study in 703 cancer patients undergoing highdose chemotherapy demonstrated that prolonged troponin I elevation one month after therapy was associated with a higher decrease in LVEF and associated with higher rates of a combined adverse endpoint (cardiac death, pulmonary edema, overt heart failure, LVEF-reduction $>25 \%$, lifethreatening arrhythmias) than isolated troponin I elevation immediately after therapy (64). In a small prospective study, serial troponin I measurements in breast cancer patients identified that the velocity of troponin I increase during chemotherapy was higher in patients who develop clinically apparent heart failure or a decline of LVEF $<50 \%$ during a three-years follow-up (68). Recent studies suggested that combined echocardiographic characterization and troponin measurement could be beneficial for prediction of cardiotoxicity. Global longitudinal strain (GLS) is increasingly used as a parameter for myocardial function (55). Combination of troponin $\mathrm{T}$ and myocardial GLS measurement increased sensitivity from $86 \%$ to $93 \%$ in a small group of patients treated with the anthracycline epirubicin. Either impaired GLS or elevated troponin $\mathrm{T}$ were associated with a 15 -fold risk for $\mathrm{LV}$ dysfunction $(54,69,70)$.

A role of serum troponin level in predicting response to heart failure therapy in manifest cardiotoxicity and a benefit of serum troponin in biomarker-guided therapy have been shown in prospective studies $(63,67,71)$. The association of troponin and LVEF recovery was tested in 251 patients receiving trastuzumab. LVEF recovery was less frequent in patients with elevated troponin I than in troponin-negative patients in patients (35\% vs. 100\%) (67). Troponin I as a biomarker-guided preventive therapy of cardiotoxicity has been evaluated in a cohort of 473 cancer patients receiving different regimes of high dose chemotherapy (63). One hundred and fourteen (26\%) patients with elevated peak troponin I $>0.07 \mathrm{ng} / \mathrm{mL}$ were characterized "at risk" for cardiotoxicity. The identified patients were randomized to receive to ACE inhibitor enalapril or no medication for one year. Control subjects showed a significant and sustained reduction in LVEF while LVEF in enalapril-treated patients 
remained constant (63). However, a recent prospective trial could not show a benefit of a troponin-guided strategy compared to troponin-independent preventive administration of enalapril (71).

Several prospective randomized studies have recently presented preventive and therapeutic approaches in cancer therapy-related LV-dysfunction. The OVERCOME trial (preventiOn of left Ventricular dysfunction with Enalapril and caRvedilol in patients submitted to intensive ChemOtherapy for the treatment of Malignant hEmopathies) in patients undergoing chemotherapy or autologous stem cell transplantation tested the effect of enalapril and carvedilol starting before chemotherapy for a period of six months (43). After 6 months, patients treated with enalapril and carvedilol had stable LVEF while LVEF decreased in the control group. Also, control patients had higher rates of combined death or heart failure. Highsensitive troponin I and BNP were obtained before and after chemotherapy cycles. Interestingly, both biomarkers were comparable between intervention and control group. In addition, no association was found between LVEF and troponin I or BNP level (43). The PRADA trial (PRevention of cArdiac Dysfunction during Adjuvant breast cancer therapy) aimed to investigate preventive treatment for cardiotoxicity in 130 breast cancer patients receiving anthracycline-based chemotherapy, trastuzumab (22\%) and radiotherapy (70\%). Patients were randomized to receive candesartan, metoprolol or placebo controls. Magnetic resonance imaging (MRI) guided LVEF assessment revealed a small significant higher LVEF in candesartan treated patients (1.8\% LVEF difference) while no effect using metoprolol could be seen. However, the increase of troponin in control patients was attenuated using metoprolol but not in candesartan-treated patients $(36,72)$. In a recent trial investigating "Carvedilol for Prevention of Chemotherapy-Related CardiotoxicitY" (CECCY trial), decreased serum troponin I was found at nine and 12 months in breast cancer patients undergoing anthracycline and cyclophosphamide therapy treated with carvedilol compared to placebo (73). However, the authors did not report on an association between elevated troponin I and LVEF or heart failure symptoms (73).

\section{Use of biomarkers for detection and prevention of therapy- related heart failure}

In patients with planned cardiotoxic cancer therapy, clinical examination, ECG, echocardiography with strain analysis and assessment of troponin I/T and NT-proBNP for the evaluation of baseline risk can be recommended. If baseline characterization reveals pathologic findings (ejection fraction $<50 \%$, abnormal GLS, abnormal troponin or NT-proBNP), it can be recommended to start ACE inhibitor/AT1 blocker or $\beta$ blocker therapy $(5,63,71)$. Cardiovascular risk factors and disease should be monitored and optimized (5). Management of dyslipidemia is of particular importance (74). A tendency towards an increased frequency of heart failure in breast cancer patients undergoing anthracycline treatment was observed when low-density lipoprotein (LDL) was elevated (LDL >100 m/dL) $(75)$ and patients showed lower rates of new-onset heart failure when treated with statins $(75,76)$. Therefore, early statin therapy that lowers LDL and exhibits beneficial anti-inflammatory and anti-oxidative effects can counteract the development of cardiotoxicity (74).

During therapy, echocardiography and assessment of troponin and NT-proBNP every three months can be recommended (5). When elevated troponin is found together with troponin dynamics, chest pain or ECG abnormalities, patients should be treated for suspected ACS according to ESC/AHA guidelines and coronary angiography should be initiated $(19,20,77)$.

\section{CAD and ACS}

Many cancer therapies can induce or aggravate acute and chronic injury to coronary arteries (4). Cancer itself, cancer therapy and common cardiovascular risk factors synergistically account for ACS risk (78). Malignant tumors induce a chronic inflammatory state (79). In preclinical models, cancer cells secrete inflammatory cytokines, which promote endothelial damage and accelerate atherosclerosis (79). Furthermore, malignant diseases often promote a prothrombotic milieu in the vasculature $(78,79)$. Common cardiovascular risk factors including arterial hypertension, hypercholesterolemia, smoking and history of $\mathrm{CAD}$ causes a further increase in ACS risk $(8,80)$. In addition, prolonged life expectancy of cancer patients raises their baseline CAD risk. Monitoring of blood pressure and serum LDL are recommended regularly in cancer patients (78).

Radiotherapy affects coronary arteries, valves, pericardium and myocardium (81). Patients receiving radiotherapy are at a particular risk for CAD and ACS. Enhanced expression of inflammatory cytokines following radiation has been related to the formation of 
a fibrotic and sclerotic remodeling of coronary arteries rather than canonical arteriosclerosis (82). Radiation exposure also damages endothelial cells causing them to switch to a senescent state with increased production of reactive oxygen species and adhesion molecules while nitric oxide production is severely decreased (83). These patho-mechanisms induce and accelerate development of atherosclerosis. Simultaneously, the inflammatory stimulus triggers myocardial fibrosis that can cause diastolic dysfunction eventually leading to heart failure with preserved ejection fraction $(81,84)$. Therefore, both CAD and heart failure can simultaneously develop after exposure to radiation $(8,81,84)$.

Several studies examined the role of early use of cardiac injury biomarkers in radiotherapy. Although there is evidence that left chest radiotherapy can induce increased high-sensitive troponin and creatine kinase MB (CK$\mathrm{MB})$, a convincing association between radiation-induced troponin activation and LV systolic or diastolic dysfunction has not been forwarded so far (85-87). Only limited data is available on the role of BNP in radiation-associated cardiotoxicity. Two prospective studies in breast cancer patients undergoing radiotherapy showed a significant increase in BNP plasma level at one month, six months and one year following radiation. Increased BNP levels were not associated with manifest or subclinical signs for $\mathrm{LV}$ dysfunction or onset of CAD. BNP levels were, however, related to the radiation doses $(88,89)$.

Myocardial ischemia caused by acute endothelial dysfunction and vasospasm or arterial thrombosis is a common adverse event in treatment with the fluoropyrimidine 5 -fluoruracil (5-FU). 5-FU induces endothelial injury and vasospasms due to abnormal vasoreactivity leading to manifest myocardial ischemia in up to $18 \%$ of patients typically within the early treatment phase (4). Calcium-antagonists and nitrate-derivates can be beneficial in 5-FU-associated vasospasm $(4,5,78)$. With a lower frequency, taxanes (paclitaxel, docetaxel) can induce troponin-positive vasospastic ACS (78). Cisplatin may cause arterial thrombosis in cerebral and coronary vessels. As a patho-mechanism, both induction of a procoagulant status and endothelial injury have been identified $(4,90)$. This may become clinically evident as ACS during treatment or result in accelerated development of CAD. A recent meta-analysis has shown that high dose anti-VEGF treatment with bevacizumab accounts for cardiac and cerebral ischemic events with a 4.4-fold relative risk (91). Also, tyrosine kinase inhibitors with anti-VEGF activity, especially regorafenib, also increase the risk for myocardial ischemia (52). Besides this finding, VEGF-targeting drugs have been shown to induce arterial hypertension that could contribute to longterm CAD development (4-6,8,79). This demonstrates that various drugs can contribute to ACS development. The exact risks have not been completely studied.

Diagnosis of ACS in cancer patients is challenging and often associated with an atypical clinical presentation compared to patients without underling malignant disease (92). Particularly, only $30.3 \%$ present with chest pain and only $44 \%$ show dyspnea when presenting with ACS (92). Frequency of these symptoms is lower in NSTE-ACS compared to ST-segment elevation myocardial infarction (STEMI) (92). The low occurrence of angina pectoris can be attributed to pain medication or neuropathy $(78,92)$. Therefore, it is recommended to assess cardiac biomarkers together with ECG and history of symptoms, risk factors, underlying cancer/cancer therapy according to ESC and AHA guidelines for NSTE-ACS $(19,20)$. No evidence for a 1-hour troponin approach in suspected ACS in cancer patients is available $(19,20,23,24,27)$. In cancer patients, timely assessment of hemoglobin, platelet count and coagulation tests are particularly necessary for a riskstratification when percutaneous coronary intervention (PCI) is planned $(4,8,78,93)$. Acute myocardial infarction requires PCI according to existing guidelines $(19,20,77)$. With regard to outcome, PCI in cancer patients has shown similar reperfusion rates compared to patients without cancer in the setting of acute STEMI (93). Anemia (hemoglobin $<12 \mathrm{~g} / \mathrm{dL}$ for women and $<13 \mathrm{~g} / \mathrm{dL}$ for men) served as an independent risk for all-cause mortality (93). It is recommended to determine the best possible individual interventional strategy in close interaction with the treating oncologist.

\section{Myocarditis}

Over the last three years, immune checkpoint inhibitors (ICI) have been widely implemented in treatment of advanced tumors $(94,95)$. Immune checkpoints such as cytotoxic T-lymphocyte-associated Protein 4 (CTLA-4) and programmed death 1 (PD-1) prevent an exaggerated (auto-) immune response against healthy cells but are exploited by tumor cells to prevent an anti-tumor immune response (96). ICI therapy induces a T-cell mediated immune reaction against tumor cells by blocking this anti-inflammatory mechanism. Since 2016, ICIs have been approved for bladder cancer, renal cell cancer, head and neck cancer, Hodgkin's lymphoma and Merkel cell carcinoma (96). 
The first evidence of potential involvement of immune checkpoints in maintaining cardiac integrity was found in immune checkpoint deficient mouse models (97). Fulminant heart failure has been observed in both CTLA-4 and PD-1 deficient mice $(97,98)$. In the latter, formation of antitroponin I antibodies was identified as the most likely underlying mechanism (97-99). Multiple clinical case reports of fulminant autoimmune myocarditis under ICI therapy have been published since $2016(100,101)$. Recent data show a comparably high prevalence of up to $1.14 \%$, a mortality of $36 \%$ (monotherapy) to $67 \%$ (combinational therapy) and a median onset of 17-34 days after the beginning of ICI therapy $(102,103)$. Most patients present with severe heart failure (102). Severe arrhythmias and cardiogenic shock with the need for intensive care unit treatment, inotropic medication and extracorporeal life support are common within this group of patients (101-104).

Given the fulminant nature of ICI-related myocarditis, early detection is essential for the best possible treatment. The American Society of Clinical Oncology (ASCO) has proposed a diagnostic workup (102). At baseline, ECG and troponin measurement should be conducted. Regarding the usual onset of ICI-related myocarditis within the first 6 weeks of therapy, it seems reasonable to execute weekly electrocardiogram (ECG) and troponin measurements within the first 4 weeks of treatment. Upon the onset of symptoms, it is recommended to obtain ECG, echocardiography, chest X-ray and assessment of troponin and NT-proBNP (105). Elevated troponin was found in $94 \%$ of all cases of ICI-related myocarditis while $100 \%$ of the patients showing major adverse cardiac events (MACE) had elevated troponin (102). The diagnostic value of NT-proBNP was lower with only $66 \%$ sensitivity and no changes in patients presenting with MACE (102). Measurement of total CK and CK-MB as well as myoglobin and lactate dehydrogenase are recommended since many patients will develop concurrent peripheral myositis (103).

Corticosteroid-based immunosuppressive therapy, optimum heart failure medication and permanent discontinuation of ICI-therapy has shown promising results in retrospective analyses and case reports. According to the ASCO-recommendations, high-dose steroid therapy (e.g., $1-2 \mathrm{mg} / \mathrm{kg}$ prednisone) should be administered initially. Higher initial steroid doses seem to be associated with lower discharge serum troponin. The beneficial use of mycophenolate, infliximab, intravenous immunoglobulins or antithymocyte globulin has been shown in individual cases (100-102,105).

\section{$V T E$}

Patients with cancer and cancer therapy are at risk for deep vein thrombosis (DVT) and pulmonary embolism (PE) $(106,107)$. D-dimers are small fibrin fragments present in the circulation. D-dimer level generally serves as a marker for thrombosis (108) but may be increased following a variety of conditions e.g. trauma, inflammation and cancer $(106,109)$. Therefore, increased D-dimer levels cannot confirm suspected VTE $(106,110)$.

Cancer is associated with hypercoagulable condition caused by the release of procoagulant factors and an inflammatory response (111). The incidence of VTE events is therefore significantly higher in cancer patients compared to the general population (112). The frequency of VTE varies with disease stage and the type of cancer $(107,113)$. Several cancer therapies can multiply this risk.

D-dimers interpretation is difficult in cancer patients. However, D-dimers levels were associated with poor prognosis in various malignancies independent from occurrence of VTE (109). Several studies have addressed the question whether a higher D-dimer cutoff point would increase diagnostic performance in suspected VTE $(114,115)$. Retrospective and prospective analyses have shown best diagnostic performance for $981-1,500 \mathrm{ng} / \mathrm{mL}$ D-dimer cutoff value $(114,115)$. However, a difference in survival has not been shown and the findings have not yet been externally validated $(114,115)$.

To summarize, it is recommended to utilize D-dimer level to rule out suspected VTE. Due to a low specificity, $\mathrm{D}$-dimer level is not suitable to confirm diagnosis but can be used to support diagnosis of suspected VTE when additional diagnostic measures (compression venous ultrasonography) were conducted. D-dimer level can be used to predict VTE risk and show an association with location of the clot in manifest PE.

\section{Future perspective and conclusion}

Lately, several novel potential biomarkers, e.g., nitric oxide (NO) metabolites, have been studied (116). Increased oxidative stress promotes cardiotoxicity in cancer patients treated with anthracyclines. When conversion of arginine to $\mathrm{NO}$ is impaired, alternative metabolites such as $\mathrm{N}$-monomethyl arginine (MMA) and dimethylarginine (ADMA) are synthesized causing a further inhibition of NO-synthase (116). In a prospective study in 170 breast cancer patients receiving doxorubicin \pm trastuzumab, 
the association between cardiotoxicity and plasma NO metabolites was examined. At two months, ADMA and MMA were associated with anthracycline-mediated cardiotoxicity with a hazard ratio of $2.70-3.33$ for each 1.5 -fold increase (116). These findings highlight a potential mechanistic, diagnostic and therapeutic value of NOmetabolites in cancer therapy-related cardiotoxicity.

Within the growing field of cardio-oncology, biomarkers represent a pivotal instrument for risk assessment, diagnosis and treatment monitoring of cancer-related and cancer therapy-related cardiotoxicity $(43,63,67,86,117)$. Complex interaction between cancer, cancer-therapy and cardiovascular conditions indicates the need for a multidisciplinary cardio-oncologic approach to ensure optimum treatment quality (4). While chemotherapyand radiotherapy-associated cardiotoxicity has been extensively studied, knowledge about cardiotoxic effects of modern molecularly targeted therapies remains sparse and studies characterizing these side effects are needed (49). To implement biomarkers in a standardized fashion, the establishment of guidelines and recommendations is crucial and will help to improve treatment of cardio-oncology patients.

\section{Acknowledgements}

Dr. L Michel acknowledges the following funding source: IFORES research grant from the Medical Faculty, University Duisburg-Essen, Germany.

\section{Footnote}

Conflicts of Interest: The authors have no conflicts of interest to declare.

\section{References}

1. Deutschland DSB. Häufigste Todesursachen 2015. 2017. Available online: https://www.destatis.de/ DE/ZahlenFakten/GesellschaftStaat/Gesundheit/ Todesursachen/Todesursachen.html

2. USA NCfHS. Health United States: Number of deaths for leading causes of death. 2015. Available online: https:// www.cdc.gov/nchs/fastats/leading-causes-of-death.htm

3. SEER Cancer Statistics Review, 1975-2015 [database on the Internet]. National Cancer Institute. 2018. Accessed: 06/2018. Available online: https://seer.cancer.gov/ csr/1975_2015/
4. Zamorano JL, Lancellotti P, Rodriguez Munoz D, et al. 2016 ESC Position Paper on cancer treatments and cardiovascular toxicity developed under the auspices of the ESC Committee for Practice Guidelines: The Task Force for cancer treatments and cardiovascular toxicity of the European Society of Cardiology (ESC). Eur Heart J 2016;37:2768-801.

5. Chang HM, Moudgil R, Scarabelli T, et al. Cardiovascular Complications of Cancer Therapy: Best Practices in Diagnosis, Prevention, and Management: Part 1. J Am Coll Cardiol 2017;70:2536-51.

6. Chang HM, Okwuosa TM, Scarabelli T, et al. Cardiovascular Complications of Cancer Therapy: Best Practices in Diagnosis, Prevention, and Management: Part 2. J Am Coll Cardiol 2017;70:2552-65.

7. Lenneman CG, Sawyer DB. Cardio-Oncology: An Update on Cardiotoxicity of Cancer-Related Treatment. Circ Res 2016;118:1008-20.

8. Yetis Sayin B, Oto MA. Acute Coronary Syndrome in Cancer Patients. Am J Cardiovasc Drugs 2018. [Epub ahead of print].

9. Moslehi J, Amgalan D, Kitsis RN. Grounding CardioOncology in Basic and Clinical Science. Circulation 2017;136:3-5.

10. Yancy CW, Jessup M, Bozkurt B, et al. 2013 ACCF/AHA guideline for the management of heart failure: a report of the American College of Cardiology Foundation/American Heart Association Task Force on Practice Guidelines. J Am Coll Cardiol 2013;62:e147-239.

11. Ponikowski P, Voors AA, Anker SD, et al. 2016 ESC Guidelines for the diagnosis and treatment of acute and chronic heart failure: The Task Force for the diagnosis and treatment of acute and chronic heart failure of the European Society of Cardiology (ESC)Developed with the special contribution of the Heart Failure Association (HFA) of the ESC. Eur Heart J 2016;37:2129-200.

12. Yancy CW, Jessup M, Bozkurt B, et al. 2017 ACC/ AHA/HFSA Focused Update of the 2013 ACCF/AHA Guideline for the Management of Heart Failure: A Report of the American College of Cardiology/American Heart Association Task Force on Clinical Practice Guidelines and the Heart Failure Society of America. J Card Fail 2017;23:628-51.

13. Weber M, Mitrovic V, Hamm C. B-type natriuretic peptide and $\mathrm{N}$-terminal pro-B-type natriuretic peptide Diagnostic role in stable coronary artery disease. Exp Clin Cardiol 2006;11:99-101.

14. Takase H, Dohi Y. Kidney function crucially affects B-type 
natriuretic peptide (BNP), N-terminal proBNP and their relationship. Eur J Clin Invest 2014;44:303-8.

15. McMurray JJV, Packer M, Desai AS, et al. AngiotensinNeprilysin Inhibition versus Enalapril in Heart Failure. N Engl J Med 2014;371:993-1004.

16. Felker GM, Anstrom KJ, Adams KF, et al. Effect of natriuretic peptide-guided therapy on hospitalization or cardiovascular mortality in high-risk patients with heart failure and reduced ejection fraction: A randomized clinical trial. JAMA 2017;318:713-20.

17. Hogenhuis J, Voors AA, Jaarsma T, et al. Anaemia and renal dysfunction are independently associated with BNP and NT-proBNP levels in patients with heart failure. Eur J Heart Fail 2007;9:787-94.

18. Madamanchi C, Alhosaini H, Sumida A, et al. Obesity and natriuretic peptides, BNP and NT-proBNP: mechanisms and diagnostic implications for heart failure. Int J Cardiol 2014;176:611-7.

19. Amsterdam EA, Wenger NK, Brindis RG, et al. 2014 AHA/ACC guideline for the management of patients with non-ST-elevation acute coronary syndromes: a report of the American College of Cardiology/American Heart Association Task Force on Practice Guidelines. Circulation 2014;130:e344-426. Erratum in: Circulation 2014;130:e433-4. Dosage error in article text.

20. Roffi M, Patrono C, Collet JP, et al. 2015 ESC Guidelines for the management of acute coronary syndromes in patients presenting without persistent ST-segment elevation: Task Force for the Management of Acute Coronary Syndromes in Patients Presenting without Persistent ST-Segment Elevation of the European Society of Cardiology (ESC). Eur Heart J 2016;37:267-315.

21. Westermann D, Neumann JT, Sörensen NA, et al. Highsensitivity assays for troponin in patients with cardiac disease. Nat Rev Cardiol 2017;14:472.

22. Solecki K, Dupuy AM, Kuster N, et al. Kinetics of highsensitivity cardiac troponin $\mathrm{T}$ or troponin I compared to creatine kinase in patients with revascularized acute myocardial infarction. Clin Chem Lab Med 2015;53:707-14.

23. Jaeger C, Wildi K, Twerenbold R, et al. One-hour rulein and rule-out of acute myocardial infarction using highsensitivity cardiac troponin I. Am Heart J 2016;171:92102.e1-5.

24. Mokhtari A, Borna C, Gilje P, et al. A 1-h Combination Algorithm Allows Fast Rule-Out and Rule-In of Major Adverse Cardiac Events. J Am Coll Cardiol 2016;67:1531-40.
25. Mueller C, Giannitsis E, Christ M, et al. Multicenter Evaluation of a 0-Hour/1-Hour Algorithm in the Diagnosis of Myocardial Infarction With High-Sensitivity Cardiac Troponin T. Ann Emerg Med 2016;68:76-87.e4.

26. Neumann JT, Sorensen NA, Schwemer T, et al. Diagnosis of Myocardial Infarction Using a HighSensitivity Troponin I 1-Hour Algorithm. JAMA Cardiol 2016;1:397-404.

27. Twerenbold R, Badertscher P, Boeddinghaus J, et al. 0/1-Hour Triage Algorithm for Myocardial Infarction in Patients With Renal Dysfunction. Circulation 2018;137:436-51.

28. Roos A, Bandstein N, Lundbäck M, et al. Stable HighSensitivity Cardiac Troponin T Levels and Outcomes in Patients With Chest Pain. J Am Coll Cardiol 2017;70:2226.

29. Pavo N, Raderer M, Hulsmann M, et al. Cardiovascular biomarkers in patients with cancer and their association with all-cause mortality. Heart 2015;101:1874-80.

30. Tan LL, Lyon AR. Role of Biomarkers in Prediction of Cardiotoxicity During Cancer Treatment. Curr Treat Options Cardiovasc Med 2018;20:55.

31. Volkova M, Russell R 3rd. Anthracycline cardiotoxicity: prevalence, pathogenesis and treatment. Curr Cardiol Rev 2011;7:214-20.

32. Swain SM, Whaley FS, Ewer MS. Congestive heart failure in patients treated with doxorubicin: a retrospective analysis of three trials. Cancer 2003;97:2869-79.

33. Henri C, Heinonen T, Tardif JC. The Role of Biomarkers in Decreasing Risk of Cardiac Toxicity after Cancer Therapy. Biomark Cancer 2016;8:39-45.

34. Curigliano G, Cardinale D, Suter T, et al. Cardiovascular toxicity induced by chemotherapy, targeted agents and radiotherapy: ESMO Clinical Practice Guidelines. Ann Oncol 2012;23 Suppl 7:vii155-66.

35. Lotrionte M, Biondi-Zoccai G, Abbate A, et al. Review and meta-analysis of incidence and clinical predictors of anthracycline cardiotoxicity. Am J Cardiol 2013;112:1980-4.

36. Gulati G, Heck SL, Ree AH, et al. Prevention of cardiac dysfunction during adjuvant breast cancer therapy (PRADA): a 2 × 2 factorial, randomized, placebocontrolled, double-blind clinical trial of candesartan and metoprolol. Eur Heart J 2016;37:1671-80.

37. Hamirani Y, Fanous I, Kramer CM, et al. Anthracyclineand trastuzumab-induced cardiotoxicity: a retrospective study. Med Oncol 2016;33:82.

38. Mantarro S, Rossi M, Bonifazi M, et al. Risk of severe 
cardiotoxicity following treatment with trastuzumab: a meta-analysis of randomized and cohort studies of 29,000 women with breast cancer. Intern Emerg Med 2016;11:123-40.

39. Guglin M, Hartlage G, Reynolds C, et al. Trastuzumabinduced cardiomyopathy: not as benign as it looks? A retrospective study. J Card Fail 2009;15:651-7.

40. Cardinale D, Colombo A, Bacchiani G, et al. Early detection of anthracycline cardiotoxicity and improvement with heart failure therapy. Circulation 2015;131:1981-8.

41. Vandecruys E, Mondelaers V, De Wolf D, et al. Late cardiotoxicity after low dose of anthracycline therapy for acute lymphoblastic leukemia in childhood. J Cancer Surviv 2012;6:95-101.

42. Nabati M, Janbabai G, Baghyari S, et al. Cardioprotective Effects of Carvedilol in Inhibiting Doxorubicin-induced Cardiotoxicity. J Cardiovasc Pharmacol 2017;69:279-85.

43. Bosch X, Rovira M, Sitges M, et al. Enalapril and carvedilol for preventing chemotherapy-induced left ventricular systolic dysfunction in patients with malignant hemopathies: the OVERCOME trial (preventiOn of left Ventricular dysfunction with Enalapril and caRvedilol in patients submitted to intensive ChemOtherapy for the treatment of Malignant hEmopathies). J Am Coll Cardiol 2013;61:2355-62.

44. Gottdiener JS, Appelbaum FR, Ferrans VJ, et al. Cardiotoxicity associated with high-dose cyclophosphamide therapy. Archives of Internal Medicine 1981;141:758-63.

45. Martin M, Fornecker LM, Marcellin L, et al. Acute and fatal cardiotoxicity following high-dose cyclophosphamide in a patient undergoing autologous stem cell transplantation for systemic sclerosis despite satisfactory cardiopulmonary screening. Bone Marrow Transplant 2017;52:1674-7.

46. Bronte E, Bronte G, Novo G, et al. Cardiotoxicity mechanisms of the combination of BRAF-inhibitors and MEK-inhibitors. Pharmacol Ther 2018. [Epub ahead of print].

47. Coupe N, Corrie P, Hategan M, et al. PACMEL: a phase 1 dose escalation trial of trametinib (GSK1120212) in combination with paclitaxel. Eur J Cancer 2015;51:359-66.

48. Falchook GS, Lewis KD, Infante JR, et al. Activity of the oral MEK inhibitor trametinib in patients with advanced melanoma: a phase 1 dose-escalation trial. Lancet Oncol 2012;13:782-9.

49. Knispel S, Zimmer L, Kanaki T, et al. The safety and efficacy of dabrafenib and trametinib for the treatment of melanoma. Expert Opin Drug Saf 2018;17:73-87.

50. Hall PS, Harshman LC, Srinivas S, et al. The frequency and severity of cardiovascular toxicity from targeted therapy in advanced renal cell carcinoma patients. JACC Heart Fail 2013;1:72-8.

51. Waxman AJ, Clasen S, Hwang WT, et al. CarfilzomibAssociated Cardiovascular Adverse Events: A Systematic Review and Meta-analysis. JAMA Oncol 2018;4:e174519.

52. Totzeck M, Mincu RI, Mrotzek S, et al. Cardiovascular diseases in patients receiving small molecules with antivascular endothelial growth factor activity: A meta-analysis of approximately 29,000 cancer patients. Eur J Prev Cardiol 2018;25:482-94.

53. Wang YD, Chen SX, Ren LQ. Serum B-type natriuretic peptide levels as a marker for anthracycline-induced cardiotoxicity. Oncol Lett 2016;11:3483-92.

54. Sawaya H, Sebag IA, Plana JC, et al. Early detection and prediction of cardiotoxicity in chemotherapy-treated patients. Am J Cardiol 2011;107:1375-80.

55. Sawaya H, Sebag IA, Plana JC, et al. Assessment of echocardiography and biomarkers for the extended prediction of cardiotoxicity in patients treated with anthracyclines, taxanes, and trastuzumab. Circ Cardiovasc Imaging 2012;5:596-603.

56. Lipshultz SE, Miller TL, Scully RE, et al. Changes in cardiac biomarkers during doxorubicin treatment of pediatric patients with high-risk acute lymphoblastic leukemia: associations with long-term echocardiographic outcomes. J Clin Oncol 2012;30:1042-9.

57. Gupta V, Kumar Singh S, Agrawal V, et al. Role of ACE inhibitors in anthracycline-induced cardiotoxicity: A randomized, double-blind, placebo-controlled trial. Pediatr Blood Cancer 2018:e27308.

58. Mavinkurve-Groothuis AM, Groot-Loonen J, Bellersen L, et al. Abnormal NT-pro-BNP levels in asymptomatic long-term survivors of childhood cancer treated with anthracyclines. Pediatr Blood Cancer 2009;52:631-6.

59. Sherief LM, Kamal AG, Khalek EA, et al. Biomarkers and early detection of late onset anthracycline-induced cardiotoxicity in children. Hematology 2012;17:151-6.

60. Blaes A, Konety S, Hurley P. Cardiovascular Complications of Hematopoietic Stem Cell Transplantation. Curr Treat Options Cardiovasc Med 2016;18:25.

61. Snowden JA, Hill GR, Hunt P, et al. Assessment of cardiotoxicity during haemopoietic stem cell transplantation with plasma brain natriuretic peptide. Bone Marrow Transplant 2000;26:309-13.

62. Cardinale D, Sandri MT, Martinoni A, et al. Left 
ventricular dysfunction predicted by early troponin I release after high-dose chemotherapy. J Am Coll Cardiol 2000;36:517-22.

63. Cardinale D, Colombo A, Sandri MT, et al. Prevention of high-dose chemotherapy-induced cardiotoxicity in highrisk patients by angiotensin-converting enzyme inhibition. Circulation 2006;114:2474-81.

64. Cardinale D, Sandri MT, Colombo A, et al. Prognostic value of troponin $\mathrm{I}$ in cardiac risk stratification of cancer patients undergoing high-dose chemotherapy. Circulation 2004;109:2749-54.

65. Cardinale D, Sandri MT, Martinoni A, et al. Myocardial injury revealed by plasma troponin $\mathrm{I}$ in breast cancer treated with high-dose chemotherapy. Ann Oncol 2002;13:710-5.

66. Dang CT, Yu AF, Jones LW, et al. Cardiac Surveillance Guidelines for Trastuzumab-Containing Therapy in EarlyStage Breast Cancer: Getting to the Heart of the Matter. J Clin Oncol 2016;34:1030-3.

67. Cardinale D, Colombo A, Torrisi R, et al. Trastuzumabinduced cardiotoxicity: clinical and prognostic implications of troponin I evaluation. J Clin Oncol 2010;28:3910-6.

68. Garrone O, Crosetto N, Lo Nigro C, et al. Prediction of anthracycline cardiotoxicity after chemotherapy by biomarkers kinetic analysis. Cardiovasc Toxicol 2012;12:135-42.

69. Quatromoni N, Scherrer-Crosbie M. Update on Incorporating Biomarkers with Imaging Findings for the Detection and Management of Cardiotoxicity. Curr Cardiol Rep 2018;20:67.

70. Kang Y, Xu X, Cheng L, et al. Two-dimensional speckle tracking echocardiography combined with high-sensitive cardiac troponin $\mathrm{T}$ in early detection and prediction of cardiotoxicity during epirubicine-based chemotherapy. Eur J Heart Fail 2014;16:300-8.

71. Cardinale D, Ciceri F, Latini R, et al. Anthracyclineinduced cardiotoxicity: A multicenter randomised trial comparing two strategies for guiding prevention with enalapril: The International CardioOncology Society-one trial. Eur J Cancer 2018;94:126-37.

72. Gulati G, Heck SL, Røsjø H, et al. Neurohormonal Blockade and Circulating Cardiovascular Biomarkers During Anthracycline Therapy in Breast Cancer Patients: Results From the PRADA (Prevention of Cardiac Dysfunction During Adjuvant Breast Cancer Therapy) Study. J Am Heart Assoc 2017;6.

73. Avila MS, Ayub-Ferreira SM, de Barros Wanderley MR Jr, et al. Carvedilol for Prevention of Chemotherapy-Related
Cardiotoxicity: The CECCY Trial. J Am Coll Cardiol 2018;71:2281-90.

74. Henninger C, Fritz G. Statins in anthracycline-induced cardiotoxicity: Rac and Rho, and the heartbreakers. Cell Death Dis 2017;8:e2564.

75. Seicean S, Seicean A, Plana JC, et al. Effect of statin therapy on the risk for incident heart failure in patients with breast cancer receiving anthracycline chemotherapy: an observational clinical cohort study. J Am Coll Cardiol 2012;60:2384-90.

76. Acar Z, Kale A, Turgut M, et al. Efficiency of Atorvastatin in the Protection of Anthracycline-Induced Cardiomyopathy. J Am Coll Cardiol 2011;58:988-9.

77. Ibanez B, James S, Agewall S, et al. 2017 ESC Guidelines for the management of acute myocardial infarction in patients presenting with ST-segment elevation: The Task Force for the management of acute myocardial infarction in patients presenting with ST-segment elevation of the European Society of Cardiology (ESC). Eur Heart J 2018;39:119-77.

78. Iliescu C, Tsitlakidou D, Giza DE, et al. Primary Percutaneous Coronary Interventions in Cancer Patients. Cancer Research Frontiers 2017;3:64-71.

79. Blann AD, Dunmore S. Arterial and venous thrombosis in cancer patients. Cardiol Res Pract 2011;2011:394740.

80. Onwudiwe NC, Kwok Y, Onukwugha E, et al. Cardiovascular event-free survival after adjuvant radiation therapy in breast cancer patients stratified by cardiovascular risk. Cancer Med 2014;3:1342-52.

81. Donnellan E, Phelan D, McCarthy CP, et al. Radiationinduced heart disease: A practical guide to diagnosis and management. Cleve Clin J Med 2016;83:914-22.

82. Di Maggio FM, Minafra L, Forte GI, et al. Portrait of inflammatory response to ionizing radiation treatment. J Inflamm (Lond) 2015;12:14.

83. Wang $Y$, Boerma $M$, Zhou D. Ionizing Radiation-Induced Endothelial Cell Senescence and Cardiovascular Diseases. Radiat Res 2016;186:153-61.

84. Saiki H, Petersen IA, Scott CG, et al. Risk of Heart Failure With Preserved Ejection Fraction in Older Women After Contemporary Radiotherapy for Breast Cancer. Circulation 2017;135:1388-96.

85. Zaher E, Fahmy E, Mahmoud K, et al. Assessment of the onset of radiation-induced cardiac damage after radiotherapy of breast cancer patients. Alexandria Journal of Medicine 2018. [In press].

86. Skyttä T, Tuohinen S, Boman E, et al. Troponin T-release associates with cardiac radiation doses during adjuvant 
left-sided breast cancer radiotherapy. Radiat Oncol 2015;10:141.

87. Tian S, Hirshfield KM, Jabbour SK, et al. Serum biomarkers for the detection of cardiac toxicity after chemotherapy and radiation therapy in breast cancer patients. Front Oncol 2014;4:277.

88. Palumbo I, Palumbo B, Fravolini ML, et al. Brain natriuretic peptide as a cardiac marker of transient radiotherapy-related damage in left-sided breast cancer patients: A prospective study. Breast 2016;25:45-50.

89. D'Errico MP, Petruzzelli MF, Gianicolo EA, et al. Kinetics of B-type natriuretic peptide plasma levels in patients with left-sided breast cancer treated with radiation therapy: Results after one-year follow-up. Int J Radiat Biol 2015;91:804-9.

90. Haugnes HS, Wethal T, Aass N, et al. Cardiovascular risk factors and morbidity in long-term survivors of testicular cancer: a 20-year follow-up study. J Clin Oncol 2010;28:4649-57.

91. Totzeck M, Mincu RI, Rassaf T. Cardiovascular Adverse Events in Patients With Cancer Treated With Bevacizumab: A Meta-Analysis of More Than 20000 Patients. J Am Heart Assoc 2017;6.

92. Yusuf SW, Daraban N, Abbasi N, et al. Treatment and outcomes of acute coronary syndrome in the cancer population. Clin Cardiol 2012;35:443-50.

93. Velders MA, Boden H, Hofma SH, et al. Outcome after ST elevation myocardial infarction in patients with cancer treated with primary percutaneous coronary intervention. Am J Cardiol 2013;112:1867-72.

94. Hellmann MD, Ciuleanu TE, Pluzanski A, et al. Nivolumab plus Ipilimumab in Lung Cancer with a High Tumor Mutational Burden. N Engl J Med 2018;378:2093-104.

95. Postow MA, Chesney J, Pavlick AC, et al. Nivolumab and Ipilimumab versus Ipilimumab in Untreated Melanoma. N Engl J Med 2015;372:2006-17.

96. Zhang JC, Chen WD, Alvarez JB, et al. Cancer immune checkpoint blockade therapy and its associated autoimmune cardiotoxicity. Acta Pharmacol Sin 2018;39:1693-8.

97. Waterhouse P, Penninger JM, Timms E, et al. Lymphoproliferative disorders with early lethality in mice deficient in Ctla-4. Science 1995;270:985-8.

98. Nishimura H, Okazaki T, Tanaka Y, et al. Autoimmune dilated cardiomyopathy in PD-1 receptor-deficient mice. Science 2001;291:319-22.

99. Okazaki T, Tanaka Y, Nishio R, et al. Autoantibodies against cardiac troponin I are responsible for dilated cardiomyopathy in PD-1-deficient mice. Nat Med 2003;9:1477-83.

100. Heinzerling L, Ott PA, Hodi FS, et al. Cardiotoxicity associated with CTLA4 and PD1 blocking immunotherapy. J Immunother Cancer 2016;4:50.

101.Johnson DB, Balko JM, Compton ML, et al. Fulminant Myocarditis with Combination Immune Checkpoint Blockade. N Engl J Med 2016;375:1749-55.

102. Mahmood SS, Fradley MG, Cohen JV, et al. Myocarditis in Patients Treated With Immune Checkpoint Inhibitors. J Am Coll Cardiol 2018;71:1755-64.

103. Moslehi JJ, Salem JE, Sosman JA, et al. Increased reporting of fatal immune checkpoint inhibitor-associated myocarditis. Lancet 2018;391:933.

104. Tajiri K, Aonuma K, Sekine I. Immune checkpoint inhibitor-related myocarditis. Jpn J Clin Oncol 2018;48:7-12.

105. Brahmer JR, Lacchetti C, Schneider BJ, et al. Management of Immune-Related Adverse Events in Patients Treated With Immune Checkpoint Inhibitor Therapy: American Society of Clinical Oncology Clinical Practice Guideline. J Clin Oncol 2018;36:1714-68.

106.Saar JA, Maack C. Diagnosis and management of acute pulmonary embolism. ESC guidelines 2014. Herz 2015;40:1048-54.

107. Timp JF, Braekkan SK, Versteeg HH, et al. Epidemiology of cancer-associated venous thrombosis. Blood 2013;122:1712-23.

108.ten Wolde M, Kraaijenhagen RA, Prins MH, et al. The clinical usefulness of d-dimer testing in cancer patients with suspected deep venous thrombosis. Arch Intern Med 2002;162:1880-4.

109.Ay C, Dunkler D, Pirker R, et al. High D-dimer levels are associated with poor prognosis in cancer patients. Haematologica 2012;97:1158-64.

110.Jaff MR, McMurtry MS, Archer SL, et al. Management of massive and submassive pulmonary embolism, iliofemoral deep vein thrombosis, and chronic thromboembolic pulmonary hypertension: a scientific statement from the American Heart Association. Circulation 2011;123:1788-830.

111. Falanga A, Russo L, Milesi V, et al. Mechanisms and risk factors of thrombosis in cancer. Crit Rev Oncol Hematol 2017;118:79-83.

112. Walker AJ, Card TR, West J, et al. Incidence of venous thromboembolism in patients with cancer - A cohort study using linked United Kingdom databases. Eur J Cancer 
2013;49:1404-13.

113.Abdel-Razeq HN, Mansour AH, Ismael YM. Incidental pulmonary embolism in cancer patients: clinical characteristics and outcome--a comprehensive cancer center experience. Vasc Health Risk Manag 2011;7:153-8.

114. Chen C, Li G, Liu YD, et al. A new D-dimer cutoff value to improve the exclusion of deep vein thrombosis in cancer patients. Asian Pac J Cancer Prev 2014;15:1655-8.

115.Ferroni P, Martini F, Portarena I, et al. Novel highsensitive D-dimer determination predicts chemotherapy-

Cite this article as: Michel L, Rassaf T, Totzeck M. Biomarkers for the detection of apparent and subclinical cancer therapyrelated cardiotoxicity. J Thorac Dis 2018;10(Suppl 35):S4282S4295. doi: 10.21037/jtd.2018.08.15 associated venous thromboembolism in intermediate risk lung cancer patients. Clin Lung Cancer 2012;13:482-7. 116. Finkelman BS, Putt M, Wang T, et al. Arginine-Nitric Oxide Metabolites and Cardiac Dysfunction in Patients With Breast Cancer. J Am Coll Cardiol 2017;70:152-62.

117.Pabinger I, van Es N, Heinze G, et al. A clinical prediction model for cancer-associated venous thromboembolism: a development and validation study in two independent prospective cohorts. Lancet Haematol 2018;5:e289-98. 